\title{
Challenges in Large-Scale Open Agent Mediated Economies
}

\author{
Steven Willmott ${ }^{1}$, Monique Calisti ${ }^{2}$, and Emma Rollon ${ }^{1}$ \\ ${ }^{1}$ Laboratoire d'Intelligence Artificielle, Department Informatique, \\ Swiss Federal Institute of Technology, IN (Ecublens), CH-1015 Lausanne, Switzerland. \\ \{steven.willmott, emma.rollon\}@epfl.ch \\ ${ }^{2}$ Whitestein Technologies AG, Ghotthardstrasse 50, \\ 8002 Zurich Switzerland. \\ \{mca\}@whitestein.com
}

\begin{abstract}
Technology developments such as Web Services, GRID Computing and peer-to-peer toolkits are rapidly changing the way systems deployed in public networks interact. Large-scale usage of these technologies would very likely lead to environments which have many of the features commonly considered in Agent mediated e-commerce research: large-scale, dynamic populations of automated trading systems.

This paper discusses the possibility of developing a large-scale open, agent based "economy" to act as a challenging test environment for electronic commerce solutions based on agent technology. Such an environment would allow researchers and developers to better understand the properties and characteristics of their systems whilst stopping short of deploying them in a real-economic environment. Although we describe some preliminary work in the context of the Agentcities initiative we intend this paper primarily as fuel for discussion.
\end{abstract}

\section{Introduction}

While some agent based trading systems have been successful in real world applications (see $[10,12]$ for example), a challenge often faced by researchers is how to evaluate their systems under real usage conditions - in particular when the intended environment is large-scale open systems such as future Internet environments. How effectively do the solutions developed work:

- In constantly changing environmental conditions?

- In a constantly changing heterogeneous agent population?

- When interacting in multiple heterogeneous, parallel on-line markets?

These issues apply to buyer/seller behaviour style problems (e.g. optimising auction bidding) as well as agent based work-flow and the construction of virtual organisations. All these phenomena can to some extent be studied in simulations [5] or in competition style systems which provide some openness (the Trading 
Agent Competition which runs on the Michigan Internet AuctionBot platform ${ }^{1}$ ) but there is still a significant leap between these and real commercial activity. In this paper, we discuss the possibility of developing a continually running, large-scale distributed "virtual economy" in which:

- Agents can trade with one another on the basis of an imaginary currency (some kind of token) - establishing a common currency for their economic interactions.

- Such trading / service providing agents can be deployed by anybody on the basis of agreed interfaces (ontologies, languages, protocols and so forth) for the purposes of small or large scale research/development tests.

- Existing and future agent based trading services (such as market places, auction houses or simple exchange services) could be linked to provide an increasingly heterogenous trading environment for agents.

Clearly there would be many hurdles to achieve this kind of environment in its general form and such an effort would represent a long term challenge. We believe however that simple mechanisms could be used to establish some of these properties in a relatively short time. In particular, we are motivated by the current development of the Agentcities initiative ${ }^{2}$ which is working to deploy a large-scale open network of agent platforms and services. This infrastructure may provide sufficient basis for developing a truly open virtual economic system (see Section 2.2), more generally however these aims may also be achievable by leveraging emerging technologies such as Web Services [13], GRID computing infrastructures [4] or peer-to-peer software such as JXTA [7]. Whatever its basis, such an environment would enable researchers and developers to:

- Link their automated trading systems together effectively between remote sites - making it significantly easier to develop new on-line trading experiments such as TAC and opening up the possibility of studying interactions across multiple heterogenous markets or in the context of arbitrary coordination scenarios.

- Gain a greater understanding of what future networks populated by automated trading systems might look like (potentially in terms of macro/micro economic properties).

- Develop and test prototypes in a challenging "intermediate" environment as a staging post prior to transition into future commercial environments (such as large-scale Web Services, ebXML or other eBusiness networks)

This paper covers the following topics: challenges in creating and exploiting such an environment (Section 3), steps required for realization (Section 4), description of a prototype payment services already deployed in the Agentcities network (Section 5) and future work / conclusions (Section 6 and Section 7). We begin with an overview of relevant work in the agent mediated commerce area

\footnotetext{
${ }^{1}$ http://auction2.eecs.umich.edu/

${ }^{2}$ http://www.agentcities.org/
} 
and an introduction to relevant aspects of the Agentcities initiative (Section 2). The paper is intended to be primarily discursive to stimulate discussion on the feasibility and utility of developing support for a large-scale open agent mediated economy.

\section{Background}

This section gives a brief overview of current research in the area of agent mediated e-Commerce and an introduction to the Agentcities activity.

\subsection{Agent E-commerce Research}

The research and practice of applying agent technology to facilitate commercial transactions is well established - previous editions of this workshop have seen a wide range of interesting theories and approaches proposed. In the last five years, agent technology also has increasingly been proposed as one of the most suitable approach for evolving the way on-line currently business is done [8]. Automated agents can follow specific strategies more efficiently and more flexibly, evaluate and optimise the utility of specific actions (such as bidding, offering, etc.). However, the integration of economic principles in electronic environments reveals the limitations of software instruments and the need to modify some economic mechanisms from their original formulation.

This problem is even more complex when considering open, heterogeneous and highly dynamic environments where the behaviour of other agents in the system cannot be either controllable nor predictable [6]. Two examples of work which creates a more open environment are the Trading Agent Competition and the Fish Market Auction house - each of which encourage users to build trading bots to compete against one another:

- The Trading Agent Competition (TAC) market games run over the Internet, on the Michigan Internet AuctionBot platform ${ }^{3}$, a configurable auction server that implements a diverse set of auction rules. The AuctionBot supports participation by software agents through a TCP-based agent programming interface (API). The TAC system provides developers a method to test their agents in a simulated TAC game. TAC trading agents operate within a travel shopping scenario, buying and selling goods to best serve their given travel clients. TAC scores the results based on the client's preferences for trips assembled, and net expenditures in the travel markets.

- Another interesting initiative is represented by the Fish-Market auction place [11]. This institution allows buyers to bid for boxes of fish that are presented by an auctioneer who also calls prices. The concatenation of many rounds of such negotiations constitutes a day's work at the fish market. Before selling those boxes of fish, sellers have to deliver the fish to the sellers admission scene, and buyers need to register with the market at the buyers

\footnotetext{
${ }^{3}$ http://auction2.eecs.umich.edu/
} 
admission scene. In this way, once a box of fish is sold, the buyer should take it away by passing through a buyers admission scene, while sellers can collect the payments at the sellers admission scene, after their lot has been sold.

This type of research provides valuable insights into the development of strategies in environments where agents have potentially diverse and unpredictable strategies. Another thread of work is large-scale economic simulation [5] which aims to trace the dynamics large populations of automated trading agents might display.

\section{$2.2 \quad$ Agentcities}

Agentcities is an on-going initiative to develop and exploit a large-scale test bed in which any researcher or developer can deploy their own agent-based systems and services. The objective of the initiative is to create a global open system to provide the conditions in which to test agents, services and other technologies, such as delegation, coordination, modelling of dynamics and, in particular, communication based on formal semantics.

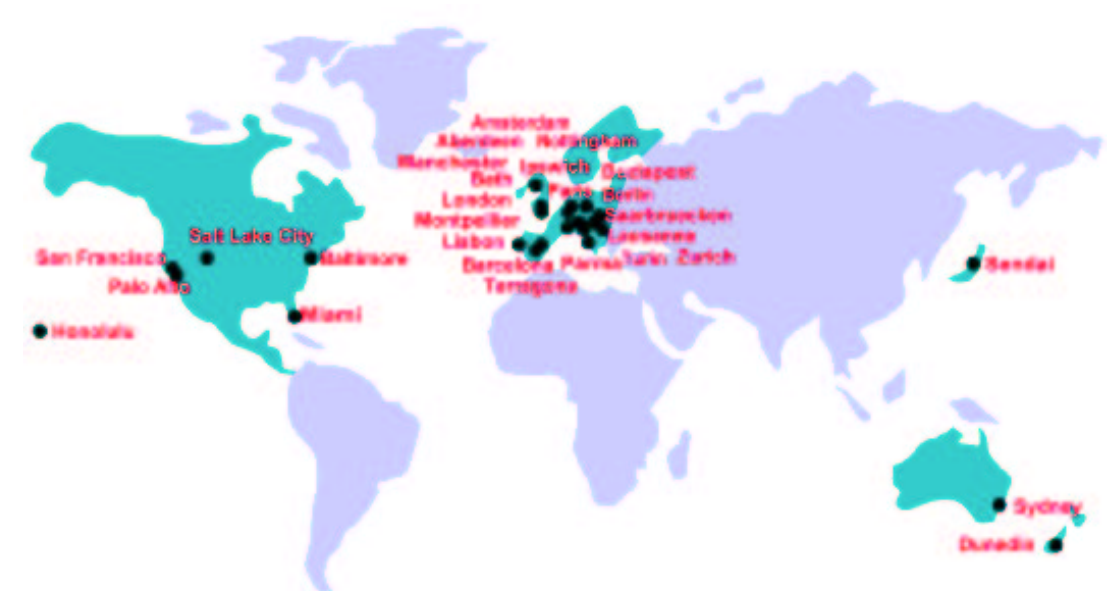

Fig. 1. Recent (Feb 2002) snapshot of the Agentcities network - showing individual agent platforms deployed around the world, the number of platforms has now risen to around 45 .

At the time of writing there are over 100 organisations involved in various Agentcities-related projects, running in 20 countries. An initial network of agent platforms based on the FIPA agent standard. ${ }^{4}$ The infrastructure is expected

\footnotetext{
${ }^{4}$ http://www.fipa.org/
} 
to evolve fast to include support for WebServices ${ }^{5}$ and other advanced interoperability technologies. There are around 45 agent platform environments now deployed and linked to the network. ${ }^{6}$ This number is expected to grow significantly as the number of funded projects increases. Most relevant for the work discussed in this paper is that the Agentcities network enables agents deployed within it to:

1. Interact with one another using standard formats and languages (HTTP, XML, FIPA-ACL, DAML+OIL).

2. Discover one another dynamically in the network.

3. Discover and access one another's services.

This is intended to provide the basis for rich interactions between agent systems in a completely open environment. The Agentcities testbed is still highly experimental but it is hoped that the infrastructure will evolve over time to improve support for complex research activities.

\subsection{Other Open Environments}

As mentioned in the introduction, there are many technology trends which look likely to enable significant increases in the amount of dynamic trading carried out in open networks. While this paper focuses on using the Agentcities environment as a basis for development, environments based on other technologies may be equally suitable.

\section{Challenges}

A number of technical and research challenges would need to be met to create a functioning economic environment.

\subsection{Research Challenges}

There research challenges both in building a large scale agent driven economy (i.e. making it possible) and in studying the behaviour of individual or collections of agents acting within it. In principle the problem is open ended. This makes it somewhat difficult to determine a scope for work. This section lists some important problems which would need to be resolved / considered.

Enabling agents to interact with diverse services clearly requires flexible communication to a level which has yet to be demonstrated. We expect agent-agent interactions (potentially based on agent languages such as FIPA-ACL) to slowly improve as they are tested/developed in the Agentcities network. There are additional problems specific to trading however, these include:

\footnotetext{
${ }^{5}$ http://www.w3c.org/2002/ws/

${ }^{6}$ A continuously updated list of which platforms are up and running can be found at http://www.agentcities.net/
} 
- Specification of semantics in the context of a particular market institution [9]. If agents are to be able to interact with multiple markets the meaning of messages in each market institution (which can be considered as a "semantic bubble" must be clearly defined.

- The rules which apply to trading in the context of a particular institution must also be clearly specified - declaring market rules in an open and machine readable way. (Note that this is related to but different from the semantics problem)

- Agents would often need to be clearly identified and traceable for many types of trading interaction. In particular it may be important to enable agents to take on long lasted (persistent) identities to study effects such as reputation in the environment.

These agent-institution issues underlie more global research issues such as:

- The dynamics of the trading environment - can price setting be regulated, controlled or understood for different types of goods.

- How can micro and macro economic indicators and controls be applied meaningfully.

These may be rather too ambitious for initial systems and perhaps overly complex to achieve. However we believe that such analysis would become increasingly important as on-line systems become more automated since it is unclear how direct and indirect control should be exercised to ensure the environment remains stable.

\subsection{Technical Challenges}

Alongside the deeper research questions there are also a number of challenging technical problems which would need to be solved before such an environment could function effectively. These include:

- Specification of interfaces between agents and payment services (potentially based on formal agent communication languages).

- Specification and deployment of banking/payment services (preferably multiple instances) to manage payments in the environment.

- Specification and development of "central bank" services to manage micro and macro-economic parameters (in particular liquidity).

- Human interfaces to monitor agent behaviour (both individual agents and the global economy).

- Security services to prevent economic breakdown due to malicious agents.

- Linking to existing real e-payment systems, real trading systems and enabling long term transition to real commercial activity.

These systems could be developed at various levels of complexity and potentially very simply at first. Note that these are additional to communication and discovery services that would need to be established. 


\section{Realisation}

This section outlines what would be necessary to create such a virtual economy. Note that much of the discussion is simplified and this is intended as a starting point only.

\subsection{Requirements}

To achieve the goals of the activity it should retain the following characteristics:

- Openness: it should be possible for anybody to create services, agents or even additional market places and financial services that interact with those already existing. ${ }^{7}$ This puts the emphasis on dealing with the issues this openness raises.

- Heterogeneity: the environment should support different types of financial interaction in different contexts (e.g. different market places for different types of goods). Variation in the type of payment mechanism may be desirable also but is perhaps a secondary consideration.

- Decentralisation: it should be possible for groups deploying systems that participate in the economy on their local machines and have them interact with those of others over the public Internet.

\subsection{Preconditions}

For the economy to function effectively, the following conditions need to be met:

1. Agreement on a virtual currency and related payment mechanisms/services.

2. There need to be a significant number of agent services deployed which are able to charge for their services in some way (simple or complex) using agreed payment mechanism(s).

3. Groups developing systems that participate in the economy would need to implement at least one service which can earn tokens.

4. Groups should also deploy systems that spend tokens in an automated way by requesting other services. (This process may need to be randomised).

5. It needs to be possible for organisations to balance the money flow between incoming tokens and outgoing tokens.

Without the incoming/outgoing element of each group tokens could get stock piled by certain services. ${ }^{8}$ The preconditions hold in addition to requirements such as communication, discovery of services and possibly security that it is assumed would be increasingly supported by the Agentcities network.

\footnotetext{
${ }^{7}$ This may be more or less difficult depending on the interfaces agreed and tools available but it should be possible.

${ }^{8}$ See Section 4.4 for comments on dealing with currency hoarding.
} 


\subsection{Spending / Making Money}

For currency to flow around the system agents would need to access on another's services. To facilitate this currency-spending cycle one could treat each research group participating in the economy (by developing agents) as running a single (agent based) company, in which some agents earn tokens for the company by selling their services on behalf of it. These tokens are subsequently used:

1. By the company to buy other component services (which provide results required to deliver the company's own service).

2. By consumer agents to access random or targeted services that are perhaps not related to the companies core business - i.e. they are spending the profits (these might be user agents for example).

In the first case service access forms part of the cash flow in the particular application developed. In the second case other mechanisms are needed to generate service access. Agents might also exercise preferences as to which services to access, or services being accessed infrequently could be placed higher in the advertising order. Clearly service access interfaces would need to be simple to reduce the overhead of implementing interactions with many services. It should be possible for agents to earn money in the Agentcities network with almost any service:

- Content Services (e.g. restaurant guide, ratings and the like) can clearly charge for content as can value added (e.g. evening organiser). These could be via subscription (and then delivering the service) or on a unit by unit basis.

- Hosting services (such as personal agent services) could charge rental fees, account setup, termination and the like. ${ }^{9}$

- Market places, payment services and the like could potentially charge commission on transactions.

- Ontology services or service directories could charge on per access or subscription basis.

\subsection{Practical Considerations}

Money is the object that makes commerce possible in a heterogeneous economy (a common mechanism for value exchange). The economy would need to ensure that:

1. The amount of money remains constant (or more or less constant) in the economy.

2. There are sufficient tokens in the economy to ensure that all systems can trade (liquidity).

3. That tokens do not get "hoarded" by agents that earn tokens but never spend them.

\footnotetext{
${ }^{9}$ This may be a little different since the service is not directly activated by an agent.
} 
There are also issues of security in transactions and fraud etc. but we believe these are somewhat secondary, especially in the beginning since one would probably need to "reboot" the economy once in a while. Eventually however, the experiment should also highlight the various security issues involved. Additionally steps would need to be taken to allow controls and systems to be overridden in certain instances. Some examples include:

- Agents becoming bankrupt - there should be mechanisms to kick start them again (e.g. a grant from the central bank).

- Agents hoarding tokens and precipitating a liquidity crisis (e.g. confiscation of funds above certain sustained levels).

- Bypassing payment for demonstrations scenarios to ensure that systems can be demonstrated no matter what the state of the current economy.

- Regularly "rebooting" the economy may initially be necessary in particular if payment systems, bank services and security systems are initially buggy or weak.

\subsection{More advanced Economic measures}

While basic payments would already provide for currency flow and the opportunity for a great deal of testing in the flexibility of agent systems interacting in multiple markets it may then be possible to further extend the model with micro and macro economic market regulation using standard means such as interest rates, exchange rates (multiple currencies), taxation, inflation and the like.

\section{Agentcities Prototype Bank Service}

This section presents a prototype set of banking services which have been implemented and deployed in the Agentcities network. The objectives of this work in the context of the European Commission funded Agentcities.RTD project are to:

- Provide as utility service for exploring the creation of dynamic value chains using agent-based interoperable services - providing a substrate for trading interactions between diverse components being developed (market places, security services, reasoning engines, diverse information services and personal agents).

- Motivate work on the integration of security services (such as trust and authentication services) into open agent environments.

- Support payments at a high enough level of abstraction to make it possible to experiment with multiple different payment schemes without dealing with the intricacies of individual technologies.

The prototype service uses the standard communication mechanisms specified for FIPA agents (FIPA-ACL [2] and FIPA-SL language [3]) as well as a DAML+OIL [1] ontology can be found on-line at http://www.lausanne.agentcities.net/. 
The Agents providing the services have recently been launched on the Lausanne Agentcities platform (see http://www.lausanne.agentcities.net) and can be accessed directly through the network.

\subsection{Agents Roles}

Two main types of agents are implemented in the e-banking service application covering and/or offering different roles and services during financial transactions.

1. Bank Agent (BA) is acting on behalf of a banking institute. Every BA offers mainly two kind of services: 1) Account management service that includes the open account, close account and list account information operations, 2) Electronic payment service in which the transfer of funds between two accounts is performed.

2. Personal Agent (PA) can be considered as a personal assistant that can act on behalf of final end users and use the services offered by the other agents.

\subsection{Agents Interaction Protocols}

In the following, we list the scenarios that are developed for defining and verifying the specific mechanisms needed and offered by the different types of agents listed above.

Scenario 1: opening an account The scenario starts when a PA-X wants to open an account within a given bank. PA-X requests the BA-UBS (for example) to open an account including the information needed. The BA-UBS will send back to PA-X the result of his demand. The message below is an example of the performative that an agent acting on behalf of a bank customer would use in order to ask for the creation of a bank account. The recipient is the agent representing the virtual banking institution.

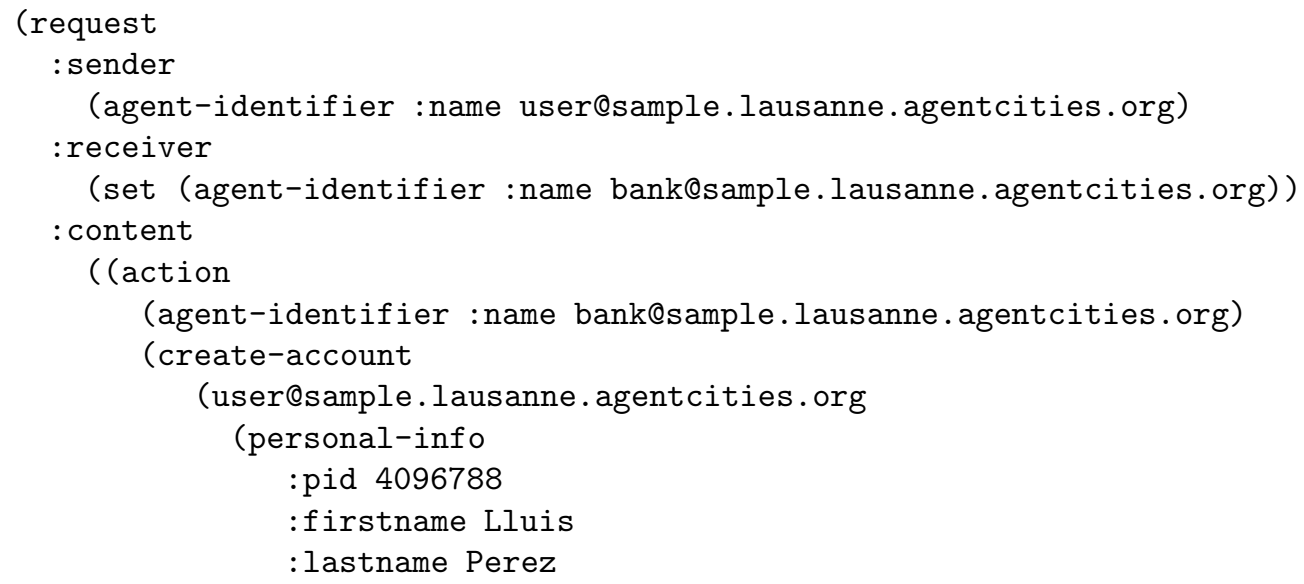




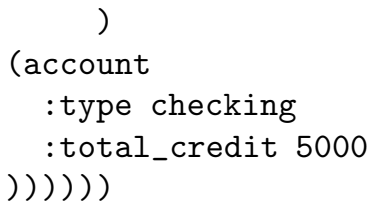

Scenario 2: getting information from an account The scenario starts when a PA-X asks for one of his accounts' information. After BA-UBS receives the query and verifies the ownership of the account, the result is sent back to de PA$\mathrm{X}$, either an error notification or the information requested. The message below shows how the agent representing an end user can ask the agent representing the banking institution to send details about the bank-account.

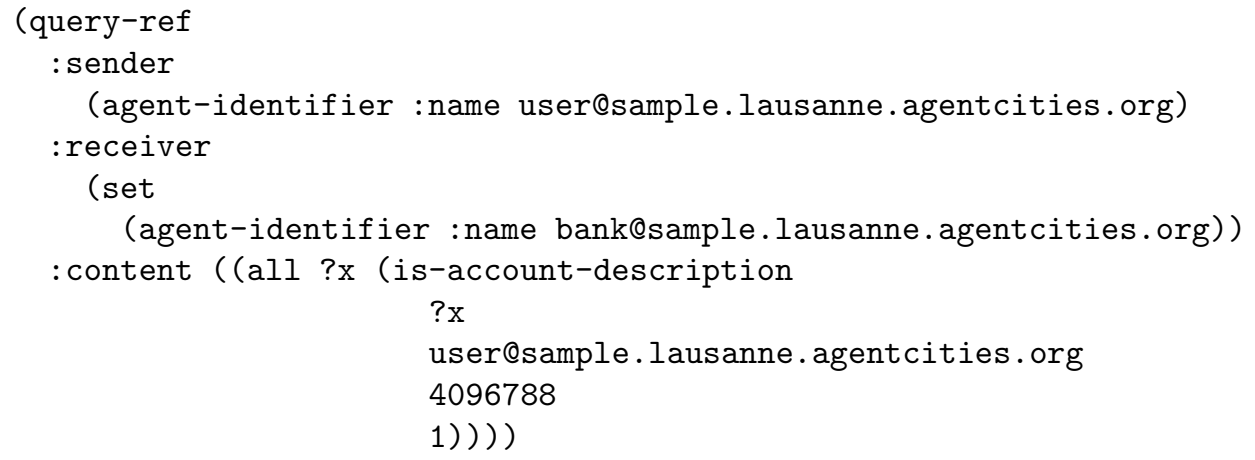

The response from the bank agent, assuming that the end user is authorised to perform the query, will be as follows:

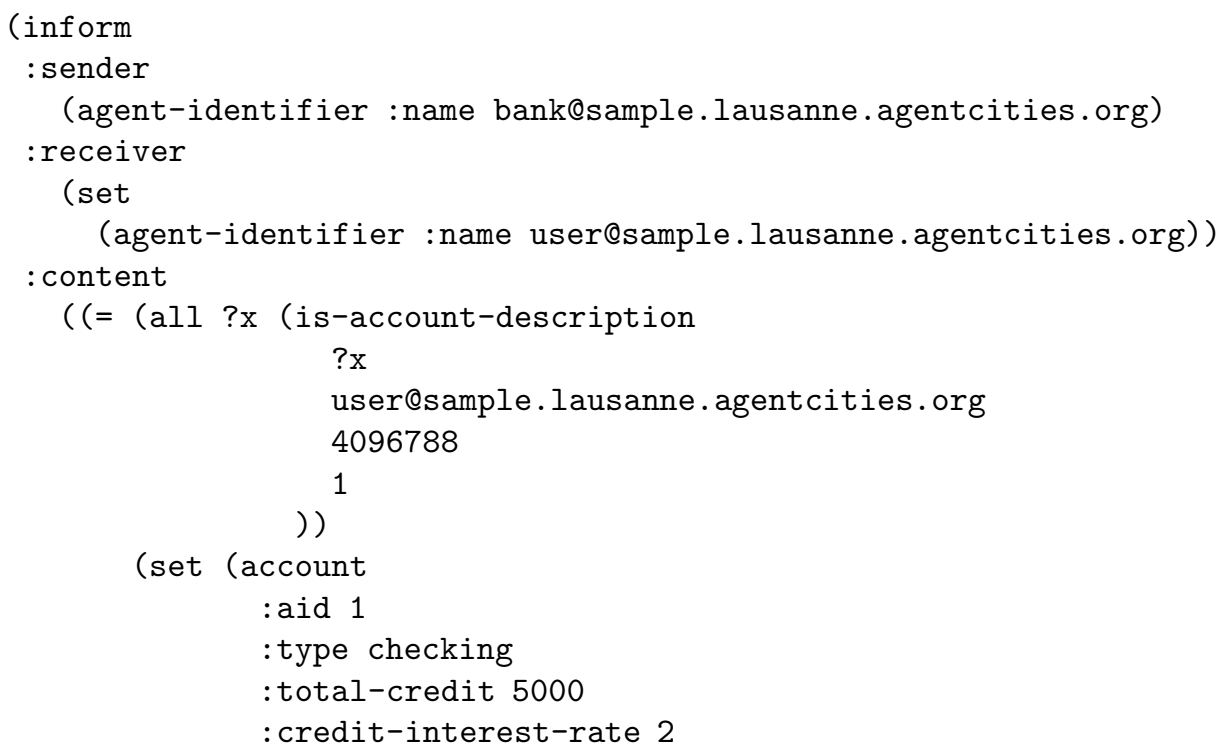




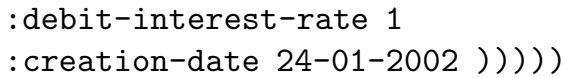

In this latter case, if the account identifier is not specified, it will be interpreted as the full list of accounts owned by the personal identifier in the bank serving the request. In the same sense, if the identifier corresponds to an employer of the bank and she has the appropriate rights, all the accounts in the bank will be listed.

Scenario 3: credit-card based payment If PA-X wishes to purchase an insurance policy from an available insurance broker (IA-CSS) one way of paying for this may be to use a credit-card style payment. PA-X sends his credit card details to IA-CSS that orders UBS bank to transfer 500 CHF from PA-X account to IA-CSS account. Both PA-X and IA-CSS have accounts with UBS (same bank agent managing both accounts) and are informed about the transaction performed. The agent's tasks are divided as follows.

- PA-to-IA negotiation: The negotiation terminates successfully if the two agents find a common agreement about the insurance policy content and its price. Depending on the specific policy that agents have been negotiation about, the final agreement can include different information and specifications. In this kind of bilateral negotiation we consider, one agent plays the role of buyer and the other agent plays the role of seller. If at the end of the negotiation an agreement is found, the PA (buyer) has to pay the IA (seller) and the seller has to supply the service to the buyer. The payment can be accomplished by finally interacting with the BA.

- The PA has to pay IA: In our case, the Insurance Agent gets the account money transfer information of the Personal Agent and asks the BA to transfer the right funds to the right account. The BA knows from the account money transfer information provided by the Insurance Agent if the transfer will be an intra or inter bank transfer (even it does not have to determine that, it just has to submit the request to the bank and the bank system will process the request accordingly). As we said before, for the moment the same bank manages both accounts.

- The BA verifies if PA's account has enough money to make the payment. If this is the case, the BA transfers the money from the buyer's account to the seller's one and notifies either the PA and the BA about the transaction. In case there is not enough money in the buyer's checking account, the BA notifies the Insurance Agent.

\subsection{Summary and Possible Extensions}

While the current bank services are very simple and still require extensive testing they do demonstrate that it is possible to represent a first step towards developing the robust services that would be required to support an effective agent economy. On-going work includes: 


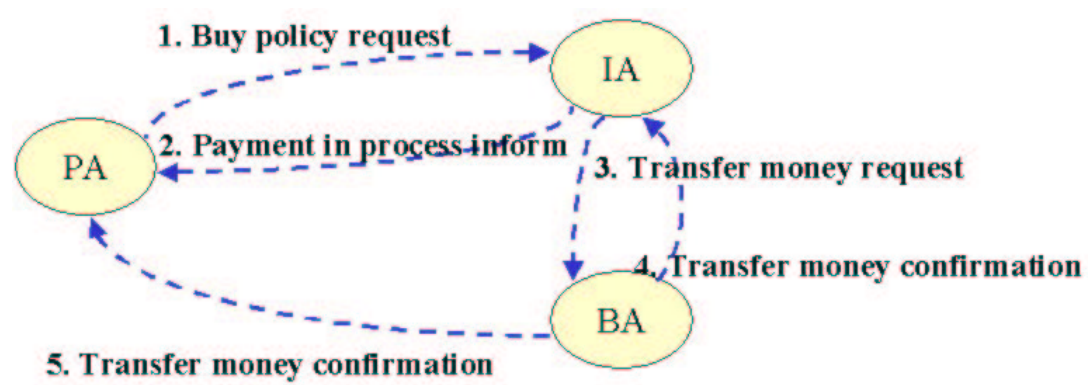

Fig. 2. Nested interactions involving IA, BA and PA

- Refinement of agent interfaces and payment functionality.

- Addition of Web accessible interfaces (to enable owners of agents using the banking services to access account information).

- Specification and development of an example "central bank" to provide an idea of how global economic trends might be followed.

- Ensuring interoperability with market place and content services being developed in the European Union 5th Framework funded Agentcities.RTD project. ${ }^{10}$

- Integration with prototype security services (to provide authentication for example).

- Addition of bank-bank interfaces to enable third parties to develop different types of payment services.

- Integration with or modelling of payment services supported by real financial institutions.

Hopefully the prototype service (and associated ontologies, protocols etc.) will provide a basis for establishment of other heterogenous trading experiments and a suitable model for developing links to real payment systems.

\section{Perspectives on the Future}

Much of the challenge related to creating an open agent economy is related to coordination of organisations interested in participating and in particular:

- Agreement on interfaces and mechanisms for payments / economic activity in the environment.

- Agreement on service instances to be developed and deployed for testing.

- Development of motivating scenarios for economic activity in the network.

With discussion on these aspects it would hopefully be possible to sketch out the scale of work needed and expected benefits. While some initial work is being carried out in the Agentcities.RTD EU funded project we hope that this will

$\overline{{ }^{10} \mathrm{http}: / / \mathrm{www}}$.agentcities.org/EURTD/ 
be extended beyond the confines of the project to address some of the deeper research/technical challenges. In particular we hope that this paper will also contribute to discussions both at the AMEC workshop and in the Agentcities Business Process Infrastructure Working Group. ${ }^{11}$

\section{Conclusions}

Web Services, ebXML and other technologies look set to transform on-line environments with heterogenous, autonomous, interacting software services - creating the possibility of increasingly interconnected electronic markets accessible to automated software systems. While existing research tools such as simulations, targeted trading competitions and analysis of real buying behaviour are crucial to understanding this "new world", it is likely to become more and more important for researchers/developers to be able to test their systems in open, heterogeneous environments.

One possible way to build knowledge in this area is to attempt to establish a common on-line economy which can be linked into existing and future trading systems. This could potentially be achieved by:

- Agreeing upon standard payment/interaction mechanisms to use in Agent trading environments (for example a common currency, protocols and the like).

- Deploying agent-based systems able to use such payment systems during their interactions.

- Managing micro/macro economic factors in the environment (or small parts of the environment) to simulate the monetary constraints in economic systems.

The engineering challenge of both constructing the necessary services (payments services, banks and so forth) and developing agents able to interact in an on-line environment (such as Agentcities, WebServices networks or P2P networks) are however non-negligible - so it remains to be seen how much benefit such an environment might bring.

In summary therefore, we hope to start discussion on how to make this feasible, what requirements would be and how the goals can be achieved.

\section{Acknowledgements}

The research described in this paper is partly supported by the European Commission funded Agentcities.RTD project, reference IST-2000-28385. The opinions expressed in this paper are those of the authors and are not necessarily those of the Agentcities.RTD project partners.

11 http://www.agentcities.org/ 


\section{References}

1. DAML. Darpa Agent Markup Language: DAML+OIL specification v 1.7. Technical report, DAML Project, Onto-Knowledge project, 2001.

2. FIPA. FIPA ACL Message Structure Specification (00061). Technical report, Foundation for Intelligence Physical Agents, 2000.

3. FIPA. FIPA SL Content Language Specification (00008). Technical report, Foundation for Intelligence Physical Agents, 2000.

4. Global Grid Forum. Grid Computing Initiative, 2002.

5. Amy R. Greenwald and Jeffrey O. Kephart. Shopbots and pricebots. In Agent Mediated Electronic Commerce (IJCAI Workshop), pages 1-23, 1999.

6. Amy R. Greenwald and Peter Stone. Autonomous bidding agents in the trading agent competition. IEEE Internet Computing, 5(2):52-, 2001.

7. Project JXTA. Project JXTA: An Open Innovate Collaboration Architecture (White Paper), 2002.

8. Hyacinth S. Nwana, Jeff Rosenschein, Tuomas Sandholm, Carles Sierra, Pattie Maes, and Rob Guttmann. Agent-mediated electronic commerce: Issues, challenges and some viewpoints. In Katia P. Sycara and Michael Wooldridge, editors, Proceedings of the 2nd International Conference on Autonomous Agents (Agents'98), pages 189-196, New York, May 9-13, 1998. ACM Press.

9. J. Pitt and A. Mamdani. A Protocol Based Semantics for and Agent Communication Language. In Proceedings of the Sixteenth International Joint Conference on Artificial Intelligence (IJCAI'99), pages 486-491. Morgan Kaufmann, 1999.

10. N. Radjou, L. Orlov, and T. Nakashima. Adaptative agents boost supply network flexibility. Techstrategy, Forrester, 2002.

11. J. Rodriguez-Aguilar, F. Martin, P. Noriega, P. Garcia, and C. Sierra. Towards a tesbed for trading agents in electronic auction markets. AI Communications 11 5-19., 1998.

12. Tuomas Sandholm. Agents in electronic commerce: Component technologies for automated negotiation and coalition formation. Autonomous Agents and MultiAgent Systems, 3(1):73-96, March 2000.

13. W3C. W3C Web Services Activity, 2002. 